\title{
Extracorporeal Shock Wave Therapy for Painful Chronic Neurogenic Heterotopic Ossification After Traumatic Brain Injury: A Case Report
}

\author{
Yong Min Choi, MD, Seok Hyun Hong, MD, Chang Hyun Lee, MD, \\ Jin Ho Kang, MD, Ju Sun Oh, MD
}

Department of Rehabilitation Medicine, Seoul Medical Center, Seoul, Korea

\begin{abstract}
Neurogenic heterotopic ossification (NHO) is a process of benign bone formation and growth in soft tissues surrounding major synovial joints and is associated with central nervous system (CNS) injuries. It is a common complication in major CNS injuries, such as traumatic brain injury, spinal cord injury, and stroke. Here, we report the case of a 72-year-old male, who experienced a traumatic brain injury and painful chronic NHO around the left hip joint. Three applications of extracorporeal shock wave therapy (ESWT) were administered to the area of NHO, which resulted in pain relief and an improvement in the loss of motion in the left hip joint. Improvements were also noted in walking performance and activities of daily living, although the size of NHO remained unchanged. Therapeutic effects of ESWT lasted for 12 weeks.
\end{abstract}

Keywords Heterotopic ossification, Extracorporeal shock wave therapy, Brain injury

\section{INTRODUCTION}

Neurogenic heterotopic ossification (NHO) is a common complication in major central nervous system injuries, such as traumatic brain injury (TBI), spinal cord injury, and stroke [1]. It is a localized and progressive formation of pathological ectopic bone in soft tissues at the major synovial joints. Significant morbidity can occur at

Received April 9, 2014; Accepted August 18, 2014

Corresponding author: Ju Sun Oh

Department of Rehabilitation Medicine, Seoul Medical Center, 371-6, Sinnae-dong, Jungrang-gu, Seoul 131-130, Korea

Tel: +82-2-2276-8640, Fax:+82-2-2276-7486, E-mail: sunnywind78@ gmail.com

(c) This is an open-access article distributed under the terms of the Creative Commons Attribution Non-Commercial License (http://creativecommons. org/licenses/by-nc/3.0) which permits unrestricted noncommercial use, distribution, and reproduction in any medium, provided the original work is properly cited.

Copyright $\odot 2015$ by Korean Academy of Rehabilitation Medicine the site of NHO, including pain and loss of motion (LOM), and can interfere with activities of daily living and reduce the quality of life. Therefore, treatment of NHO is important for improved rehabilitation.

Extracorporeal shockwave therapy (ESWT) has been shown to be an effective method of treating both acute and chronic soft-tissue painful inflammations [2]. The mechanism of ESWT is not fully understood, but it may work via anti-inflammatory mechanism [3] and tissue regeneration with neovascularization [4] and reduction of neurogenic inflammation [5].

We experienced a case with chronic painful NHO after TBI. As a mature NHO, intense inflammation was gone. Nevertheless, the severe pain had been preserved in the affected area and intractable. We thought soft tissues around NHO were compressed by calcifying mass and these mass-effects led to local ischemic injury and ne- 
crosis. Progression of NHO is enough to compress nearby neurovascular structures and thereby cause hypoxic tissue damage $[6,7]$.

We hypothesized that the recovery of soft tissue injury by mass-effects around NHO can improve the intractable pain and expected ESWT would be a useful method to enhance tissue regeneration via several mechanisms mentioned above.

To the best of our knowledge, there are no prior reports regarding the use of ESWT as a treatment for chronic painful NHO in Asian patients. We herein report this case with a review of the literature.

\section{CASE REPORT}

A 72-year-old male with no notable past medical history except diabetes and hypertension was in a pedestrian traffic accident on October 13, 2012. Craniectomy and cranioplasty were performed to treat the resulting TBI. In June 2013, he was diagnosed with NHO and etidronate was administrated for four months in a previous rehabilitation unit. Serum alkaline phosphatase (sALP) levels were almost normalized and no further progression of NHO was noted in follow-up radiography (Fig. 1). The administration of etidronate was stopped. However, he continued to experience pain in his left hip joint, so celecoxib was prescribed. At the time of admission to our rehabilitation unit on December 20, 2013, he was suffering from severe chronic pain and LOM in the affected joint without swelling or heatness. Initial sALP levels were 192 IU/L and follow-up sALP level was almost unchanged. The size of NHO had not progressed in radiography. He could not sit upright in a wheelchair due to pain and he could barely sit in a semi-recline position for 10 minutes. The modified Ashworth Scale was grade 0 for all joints except the left hip, which was not assessed due to pain. When pain levels were measured on the visual analogue scale (VAS), the initial VAS was 8 and intermittently 10. Passive range of motion of the left hip was restrictively measured using a goniometer in a supine position due to pain (Table 1). The manual muscle test score was grade III for all extremities.

He could walk using a walker. Gait speed was evaluated using the 6-minute walk test (6MWK) and gait endur-
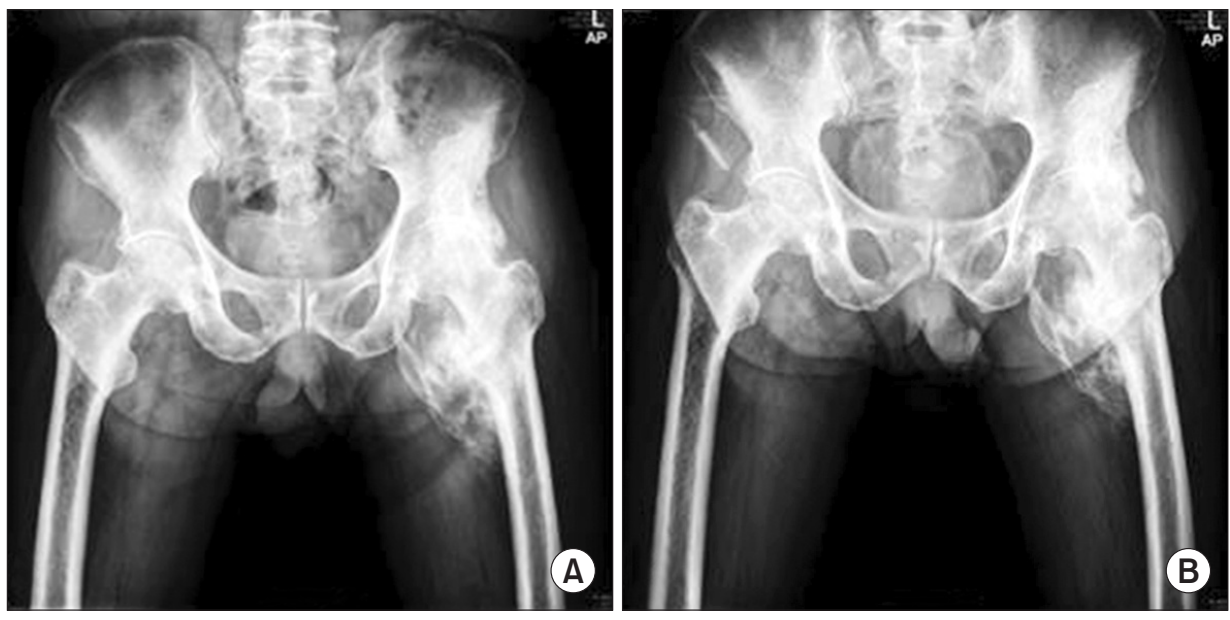

Fig. 1. (A) Radiograph of the hip showed before extracorporeal shock wave therapy (ESWT). (B) Radiograph of the hip showed after ESWT.

Table 1. Passive ROM of the left hip joint before and after ESWT

\begin{tabular}{|lcccc}
\hline Passive ROM of the left hip & Before ESWT $\left(^{\circ}\right.$ ) & After ESWT $\left(^{\circ}\right)$ & 4 weeks after ESWT $\left(^{\circ}\right.$ ) & 12 weeks after ESWT $\left(^{\circ}\right.$ ) \\
\hline Flexion & 30 & 70 & 80 & 80 \\
\hline Extension & 0 & 0 & 0 & 5 \\
\hline Abduction & 20 & 20 & 20 & 25 \\
Adduction & 20 & 20 & 20 & 20 \\
\hline Internal rotation & 50 & 50 & 50 & 50 \\
\hline External rotation & -5 & 0 & 0 & 0 \\
\hline
\end{tabular}

ROM, range of motion; ESWT, extracorporeal shock wave therapy. 
ance, which was assessed on a 70-m track in our physical therapy treatment room, was evaluated by the distance that the patient could walk before stopping due to fatigue or pain. The time taken to complete the first lap of this track during the gait endurance test was also recorded. The initial time for the $6 \mathrm{MWK}$ was 17 seconds. The distance walked in the gait endurance test was $72 \mathrm{~m}$, with the patient stopping due to hip joint pain. The time taken to walk the first $70 \mathrm{~m}$ in the gait endurance test was 272 seconds (Table 1).

Celecoxib was discontinued, and 1 week later ESWT was applied to the anterior aspect of the left hip using Dornier AR2 (Dornier MedTech, Wessling, Germany) (Fig. 2) guided by ultrasound (Fig. 3A). Each application of ESWT involved 4,000 shocks delivered at a rate of 3 $\mathrm{Hz}$ and had an energy flux density (EFD) from 0.056 to $0.068 \mathrm{~mJ} / \mathrm{mm}^{2}$. ESWT was administered once a week for 3 weeks. Physical modalities were not performed.
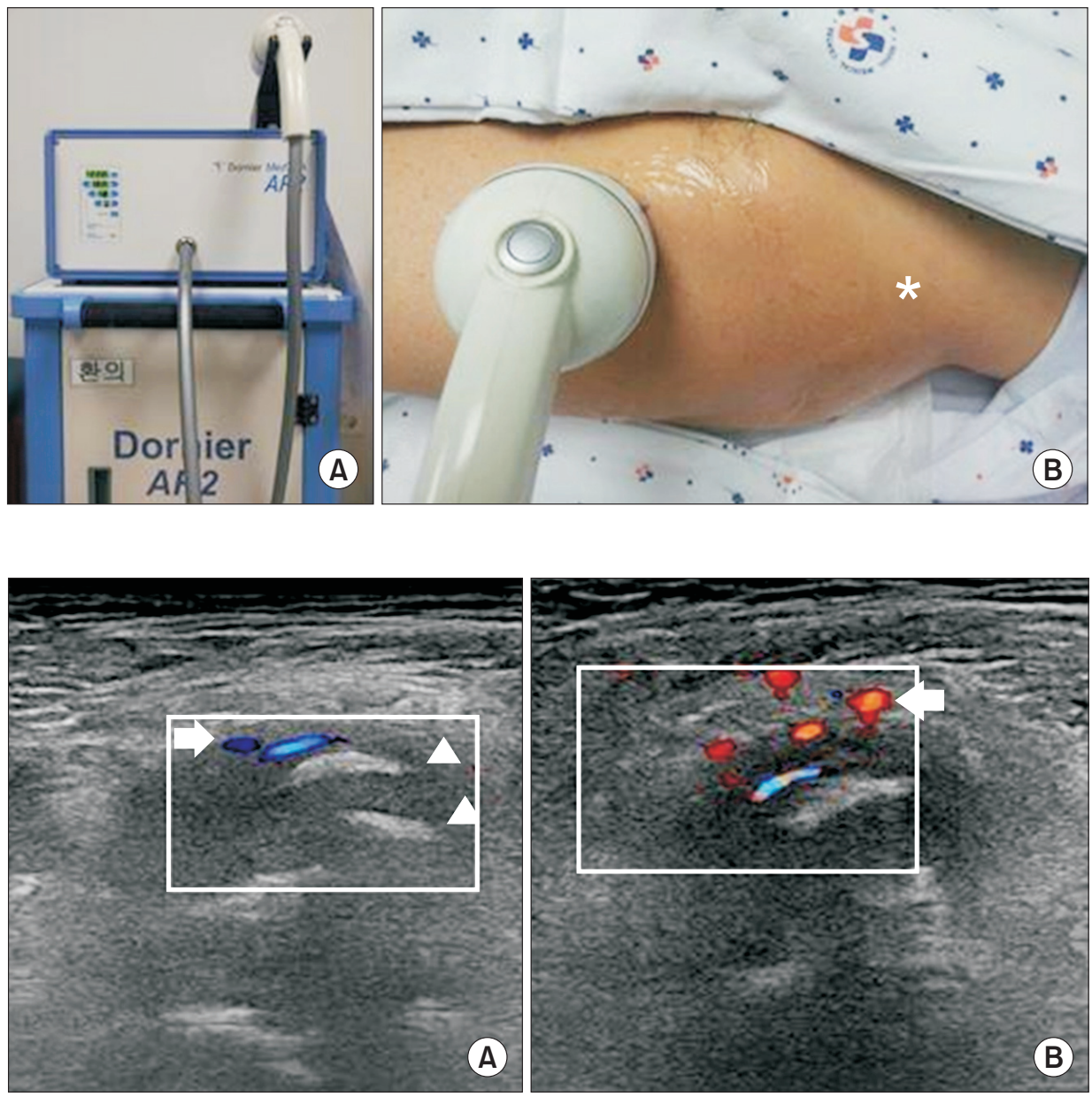

Fig. 2. (A) Dornier AR2 (Dornier MedTech, Wessling, Germany). (B) Extracorporeal shock wave therapy was applied below the anterior superior iliac spine (asterisk).

Fig. 3. (A) Transverse ultrasound image taken before extracorporeal shock wave therapy (ESWT) showed multiple hyperechoic foci with no shadowing (arrowhead), vascularity was presented in the color Doppler image (arrow). (B) Blood supply was increased in the color Doppler image (arrow) 4 weeks after ESWT.

Table 2. Measurements of clinical outcome before and after ESWT

\begin{tabular}{|lcccc}
\hline \multicolumn{1}{c}{ Clinical outcome } & Before ESWT & After ESWT & 4 weeks after ESWT & 12 weeks after ESWT \\
\hline VAS & $8-10$ & 3 & 3 & 3 \\
6-minute walk test (sec) & 17 & 15 & 13 & 14 \\
70 m travel time (sec) & 272 & 195 & 198 & 203 \\
Maximum walking distance (m) & 72 & 140 & 153 & 203 \\
sALP (IU/L) & 192 & 172 & 172 & 186 \\
Sitting time in a wheelchair (min) & 10 & 60 & 90 & 120 \\
\hline
\end{tabular}

ESWT, extracorporeal shock wave therapy; VAS, visual analogue scale; sALP, serum alkaline phosphatase. 
After ESWT, the passive flexion angle of the left hip joint was $70^{\circ}$ (Table 1 ), the sALP level was $172 \mathrm{IU} / \mathrm{L}$, and the VAS was 3. The duration for which he could sit in a wheelchair was 60 minutes, and he was able to sit comfortably and raise his left foot onto a footrest. The time for the $6 \mathrm{MWK}$ was 15 seconds, the distance walked in the gait endurance test was $140 \mathrm{~m}$, and the time taken to walk the first $70 \mathrm{~m}$ in the gait endurance test was 195 seconds (Table 2). Radiographs were similar to those obtained pre-ESWT (Fig. 1).

Four weeks after ESWT, sALP levels and VAS were maintained and other outcomes further improved (Tables 1, 2). Color Doppler images obtained 4 weeks after ESWT showed an increase in vascularization around the NHO, but no change in the hyperechoic foci (Fig. 3B). The therapeutic effects were maintained at 12 weeks after ESWT (Table 1). The patient did not request any NSAIDs after ESWT. No complications such as edema, hematoma, dizziness, and paresthesia were noted.

\section{DISCUSSION}

The most common clinical findings in NHO are LOM and peri-articular swelling due to interstitial edema of soft tissues around the affected sites [1]. NHO, especially after TBI, is not prone to regression and is hard to treat [8]. The growth of NHO results in the mass-effect, compression of the vessels and nearby peripheral nerves [6]. In this case, NHO was already relatively advanced when etidronate was administered. The NHO was large and would likely have resulted in a mass-effect that was aggravated as time passed. This would have damaged the soft tissues surrounding the NHO through hypoxic injury, inflammatory reactions and tissue necrosis, resulting in pain [7]. The mass-effect likely also triggered sequential inflammatory tissue damage and neurogenic inflammation.

Shockwaves with a low EFD can induce the ingrowth of neo-vessels and tissue proliferation associated with the early release of angiogenesis-related factors including nitric oxide (NO) and vascular endothelial growth factor $[3,4]$. The neovascularization may lead to improved blood supply and play a role in tissue regeneration. In our case, vascularity around the NHO was increased in the color Doppler images taken 4 weeks after ESWT (Fig. 3B). The local blood supply increased by ESWT around the NHO may lead to restoration of oxygen, growth factor and nutrients and thus cause the hypoxic tissue to return to aerobic metabolism $[4,6,7]$.

Shockwaves with a low EFD are also able to regulate the inflammatory cascade by modulating NO complex in soft tissues [3]. ESWT may induce a significant increase in NO and a decrease in pro-inflammatory substances, such as nuclear factor kappa B. NO may exert either an excitatory or an inhibitory effect on neuronal transmission [9] and ESWT may reduce the calcitonin gene-related peptide, considered as the main excitatory nociceptive transmitter [5,9]. Also, Wess [10] suggested that ESWT may reorganize pathologic memory giving rise to chronic pain, thus resulting in pain relief. These may explain competent management of chronic painful NHO after ESWT without the diminution in NHO (Fig. 1). Flexion of the left hip joint was particularly improved. We thought the reason was related to the location of NHO. Because NHO occurred in the anterior portion of the left hip joint, more relative limitation of flexion in the left hip joint would occur by mass-effect and the flexion of the left hip joint would significantly improve after ESWT. Also, he had progression in gait speed, gait distance and gait performance. The number of stops caused by pain was reduced during walking. Therefore, the participation in exercise improved and the total amount of exercise were increased.

The study has a number of limitations. An electrodiagnostic study was not performed to confirm nerve injuries around the area of NHO. However, the area of NHO was located a distance from the femoral or the sciatic nerve and no paresthesia was noted along cutaneous branches of their nerves. We did not check the isoenzyme of ALP, but sALP levels were not noticeably different after ESWT. The effects of passive range of motion exercise may have influenced the improvements observed. However, these exercises had been performed since the TBI, which were several months before the ESWT. It is therefore unlikely that the passive range of motion exercises had any effect on the improvements seen after 3 weeks of ESWT. Color Doppler images obtained 4 weeks after ESWT showed increased blood supply. However, power Doppler sonography was not performed and thus it was not possible to quantify the neovascularization. Further quantitative analysis of the neovascularization is therefore required.

In conclusion, this case suggests that ESWT may be 
an effective, safe, and easy-to-use treatment for chronic painful NHO after TBI.

\section{CONFLICT OF INTEREST}

No potential conflict of interest relevant to this article was reported.

\section{REFERENCES}

1. Sullivan MP, Torres SJ, Mehta S, Ahn J. Heterotopic ossification after central nervous system trauma: a current review. Bone Joint Res 2013;2:51-7.

2. Wang CJ. Extracorporeal shockwave therapy in musculoskeletal disorders. J Orthop Surg Res 2012;7:11.

3. Mariotto S, de Prati AC, Cavalieri E, Amelio E, Marlinghaus E, Suzuki H. Extracorporeal shock wave therapy in inflammatory diseases: molecular mechanism that triggers anti-inflammatory action. Curr Med Chem 2009;16:2366-72.

4. Wang CJ, Wang FS, Yang KD, Weng LH, Hsu CC, Huang CS, et al. Shock wave therapy induces neovascularization at the tendon-bone junction: a study in rabbits.
J Orthop Res 2003;21:984-9.

5. Takahashi N, Wada Y, Ohtori S, Saisu T, Moriya H. Application of shock waves to rat skin decreases calcitonin gene-related peptide immunoreactivity in dorsal root ganglion neurons. Auton Neurosci 2003;107:81-4.

6. Varghese G, Williams K, Desmet A, Redford JB. Nonarticular complication of heterotopic ossification: a clinical review. Arch Phys Med Rehabil 1991;72:100913.

7. van Kuijk AA, Geurts AC, van Kuppevelt HJ. Neurogenic heterotopic ossification in spinal cord injury. Spinal Cord 2002;40:313-26.

8. Sarafis KA, Karatzas GD, Yotis CL. Ankylosed hips caused by heterotopic ossification after traumatic brain injury: a difficult problem. J Trauma 1999;46: 104-9.

9. Riedel W, Neeck G. Nociception, pain, and antinociception: current concepts. Z Rheumatol 2001;60:40415.

10. Wess OJ. A neural model for chronic pain and pain relief by extracorporeal shock wave treatment. Urol Res 2008;36:327-34. 